\title{
Consensus Statement on the Terminology and Classification of Central Neck Dissection for Thyroid Cancer
}

\author{
The American Thyroid Association Surgery Working Group \\ with Participation from the American Association of Endocrine Surgeons, \\ American Academy of Otolaryngology_-Head and Neck Surgery, and American Head and Neck Society \\ Sally E. Carty, ${ }^{1, *}$ David S. Cooper, ${ }^{2}$ Gerard M. Doherty, ${ }^{3}$ Quan-Yang Duh, ${ }^{4}$ Richard T. Kloos, ${ }^{5}$ \\ Susan J. Mandel, ${ }^{6}$ Gregory W. Randolph, ${ }^{7}$ Brendan C. Stack, Jr., ${ }^{8}$ David L. Steward, ${ }^{9}$ David J. Terris, ${ }^{10}$ \\ Geoffrey B. Thompson, ${ }^{11}$ Ralph P. Tufano, ${ }^{12}$ R. Michael Tuttle, ${ }^{13}$ and Robert Udelsman ${ }^{14}$
}

Background: The primary goals of this interdisciplinary consensus statement are to review the relevant anatomy of the central neck compartment, to identify the nodal subgroups within the central compartment commonly involved in thyroid cancer, and to define a consistent terminology relevant to the central compartment neck dissection.

Summary: The most commonly involved central lymph nodes in thyroid carcinoma are the prelaryngeal (Delphian), pretracheal, and the right and left paratracheal nodal basins. A central neck dissection includes comprehensive, compartment-oriented removal of the prelaryngeal and pretracheal nodes and at least one paratracheal lymph node basin. A designation should be made as to whether a unilateral or bilateral dissection is performed and on which side (left or right) in unilateral cases. Lymph node "plucking" or "berry picking" implies removal only of the clinically involved nodes rather than a complete nodal group within the compartment and is not recommended. A therapeutic central compartment neck dissection implies that nodal metastasis is apparent clinically (preoperatively or intraoperatively) or by imaging (clinically N1a). A prophylactic/elective central compartment dissection implies nodal metastasis is not detected clinically or by imaging (clinically N0).

Conclusion: Central neck dissection at a minimum should consist of removal of the prelaryngeal, pretracheal, and paratracheal lymph nodes. The description of a central neck dissection should include both the indication (therapeutic vs. prophylactic/elective) and the extent of the dissection (unilateral or bilateral).

\section{Introduction}

$\mathbf{T}$ HE PRIMARY GOALS OF this interdisciplinary consensus statement are to review the relevant anatomy of the central neck compartment, to identify the nodal subgroups within the central compartment commonly involved in thyroid cancer, and to define a consistent terminology relevant to the central neck dissection. Uniformity in definition may lead to more uniform central neck surgery and may facilitate consistent and clear communication among

*Authors are listed in alphabetical order.

${ }^{1}$ Department of Surgery, Section of Endocrine Surgery, University of Pittsburgh School of Medicine, Pittsburgh, Pennsylvania.

${ }^{2}$ Division of Endocrinology and Metabolism, The Johns Hopkins University School of Medicine, Baltimore, Maryland.

${ }^{3}$ Department of Surgery, University of Michigan Health Systems, Ann Arbor, Michigan.

${ }^{4}$ Surgical Services, Veterans Affairs Medical Center and University of California, San Francisco, California.

${ }^{5}$ Divisions of Endocrinology, Diabetes and Metabolism and Nuclear Medicine; Departments of Internal Medicine and Radiology; The Ohio State University, The Arthur G. James Cancer Hospital and Richard J. Solove Research Institute, and The Ohio State University Comprehensive Cancer Center, Columbus, Ohio.

${ }^{6}$ Division of Endocrinology, Diabetes and Metabolism, University of Pennsylvania School of Medicine, Philadelphia, Pennsylvania.

${ }^{7}$ Massachusetts Eye and Ear Infirmary, Boston, Massachusetts.

${ }^{8}$ Department of Otolaryngology — Head and Neck Surgery, University of Arkansas for Medical Sciences, Little Rock, Arkansas.

${ }^{9}$ Department of Otolaryngology—Head and Neck Surgery, University of Cincinnati College of Medicine, Cincinnati, Ohio.

${ }^{10}$ Department of Otolaryngology—Head and Neck Surgery, Medical College of Georgia, Augusta, Georgia.

${ }^{11}$ Mayo Clinic, Rochester, Minnesota.

${ }^{12}$ Department of Otolaryngology-Head and Neck Surgery, Johns Hopkins University School of Medicine, Baltimore, Maryland.

${ }^{13}$ Department of Endocrinology, Memorial Sloan-Kettering Cancer Center, New York, New York.

${ }^{14}$ Department of Surgery, Yale University School of Medicine, New Haven, Connecticut. 
physicians involved in the management of patients with thyroid cancer.

Optimizing patient outcomes in thyroid cancer management involves balancing risk from treatment with risk from disease, and decisions about when to perform a central neck dissection are made individually. Specific recommendations regarding indications for central neck dissection are beyond the scope of this consensus statement. However, evidence-based guidelines provide published recommendations (1) that are revised and updated over time (2).

\section{Methods}

To obtain a multidisciplinary consensus statement on the terminology and classification of the central neck dissection for thyroid cancer, a surgery working group was established within the American Thyroid Association (ATA). The need for this working group was recognized by the ATA guidelines taskforce on thyroid cancer. Experts in thyroid cancer representing the fields of endocrinology, endocrine surgery, otolaryngology-head and neck surgery, and radiology were assembled by the ATA in consultation with the American Association of Endocrine Surgeons, the American Academy of Otolaryngology-Head and Neck Surgery, and the American Head and Neck Society. These experts are the authors of this article. All authors were involved in the discussion and participated in manuscript development. In addition to a review of the literature relevant to the central neck compartment anatomy, neck dissection classification and terminology, as well as central neck dissection for thyroid cancer, the authors solicited and considered recommendations from other experts within the fields of endocrinology, endocrine surgery, and otolaryngology-head and neck surgery. Articles were identified by searching MEDLINE using the following search terms: \{CENTRAL NECK DISSECTION\} or \{ANTERIOR NECK DISSECTION\} or \{CENTRAL NECK COMPARTMENT\} and \{THYROID\} and \{CANCER\} or \{CARCINOMA\}. Emphasis was placed on papers published between 1991 and 2008, following publication of the widely adopted report by Robbins et al., standardizing neck dissection terminology (3). Despite this original report and subsequent updates $(4,5)$, there remains controversy regarding the inferior extent of the central neck dissection, validity of unilateral versus bilateral central neck dissection, and inconsistent terminology regarding indications such as routine rather than therapeutic versus prophylactic/elective. The level of evidence primarily relied upon for the development of this article was expert opinion. The preliminary meeting of this group occurred at the ATA annual meeting in October 2007 with subsequent meetings in July and October 2008. Supplementing these meetings were multiple teleconferences and detailed electronic mail communications culminating in acceptance by the ATA Board of Directors. Additionally, this manuscript was also formally endorsed by the Boards of the American Academy of Otolaryngology-Head Neck Surgery, American Association of Endocrine Surgeons, and American Head Neck Society.

\section{Overview of the Central Compartment}

The central compartment is commonly referred to as level VI of the neck as described in the recent consensus update on neck dissection $(4,5)$. The superior (hyoid) and lateral (carotid artery) central compartment boundaries are easily recognized clinically, surgically, and with current imaging modalities. The inferior border has been variably defined as the sternal notch or the innominate (brachiocephalic) artery. Use of the latter as the lower border implies inclusion of the anterior superior mediastinum above the innominate artery, sometimes referred to as level VII $(4,5)$. The various neck levels and sublevels are presented schematically in Figure 1.

The central compartment has been subdivided into right and left sides by some authors or alternatively into right, median, and left by others. These distinctions have clinical utility relevant to sided vital structures such as parathyroid glands and laryngeal nerves but are somewhat arbitrary with regard to the lymphatic drainage from central neck structures such as the thyroid gland. Indeed, the distinction between the central (level VI) and lateral cervical lymph nodes, as well as among levels I-V and sublevels $\mathrm{Ia} / \mathrm{Ib}$, IIa/IIb, and $\mathrm{Va} / \mathrm{Vb}$, is based on a premise of minimizing morbidity from injury to vital anatomic structures during compartment-oriented nodal dissection rather than a belief that lymphatic drainage observes these anatomic landmarks.

\section{Detailed Central Compartment Anatomy}

\section{Boundaries}

The central neck compartment is bounded superiorly by the hyoid bone, laterally by the carotid arteries, anteriorly by the superficial layer of the deep cervical fascia, and posteriorly by the deep layer of the deep cervical fascia. Because the location of the thyroid gland is low in the neck near the thoracic inlet, the lymphatic drainage is contiguous with the anterior superior mediastinum that is accessible via a cervical approach. As a result, the inferior border of the central compartment is defined as the innominate artery on the right and the corresponding axial plane on the left (Fig. 2).

\section{Lymph nodes}

The most commonly involved central lymph nodes in thyroid carcinoma are the prelaryngeal (Delphian), pretracheal, and the right and left paratracheal nodes (Fig. 3). The paratracheal nodes may be anterior as well as posterior to the recurrent laryngeal nerves. These nodes may be found posterior to the common carotid on the right along the inferior thyroid artery because of the more ventral and medial location of the proximal carotid on the right compared with the left. The more posterior retropharyngeal and retroesophageal lymph nodes are less commonly involved. The vast majority of nodes within the central compartment exist inferior to the larynx; however, superior pole tumors may occasionally metastasize to paralaryngopharyngeal nodes deep to the sternohyoid and omohyoid muscles along the course of superior thyroid vasculature.

\section{Other structures}

Anatomic structures contained within the central compartment include the following: the larynx, hypopharynx, trachea and esophagus; endocrine glands (thyroid and parathyroid); cervical thymus; laryngeal nerves (superior and recurrent); vessels (inferior and superior thyroid arteries; inferior, middle, and superior thyroid veins). 


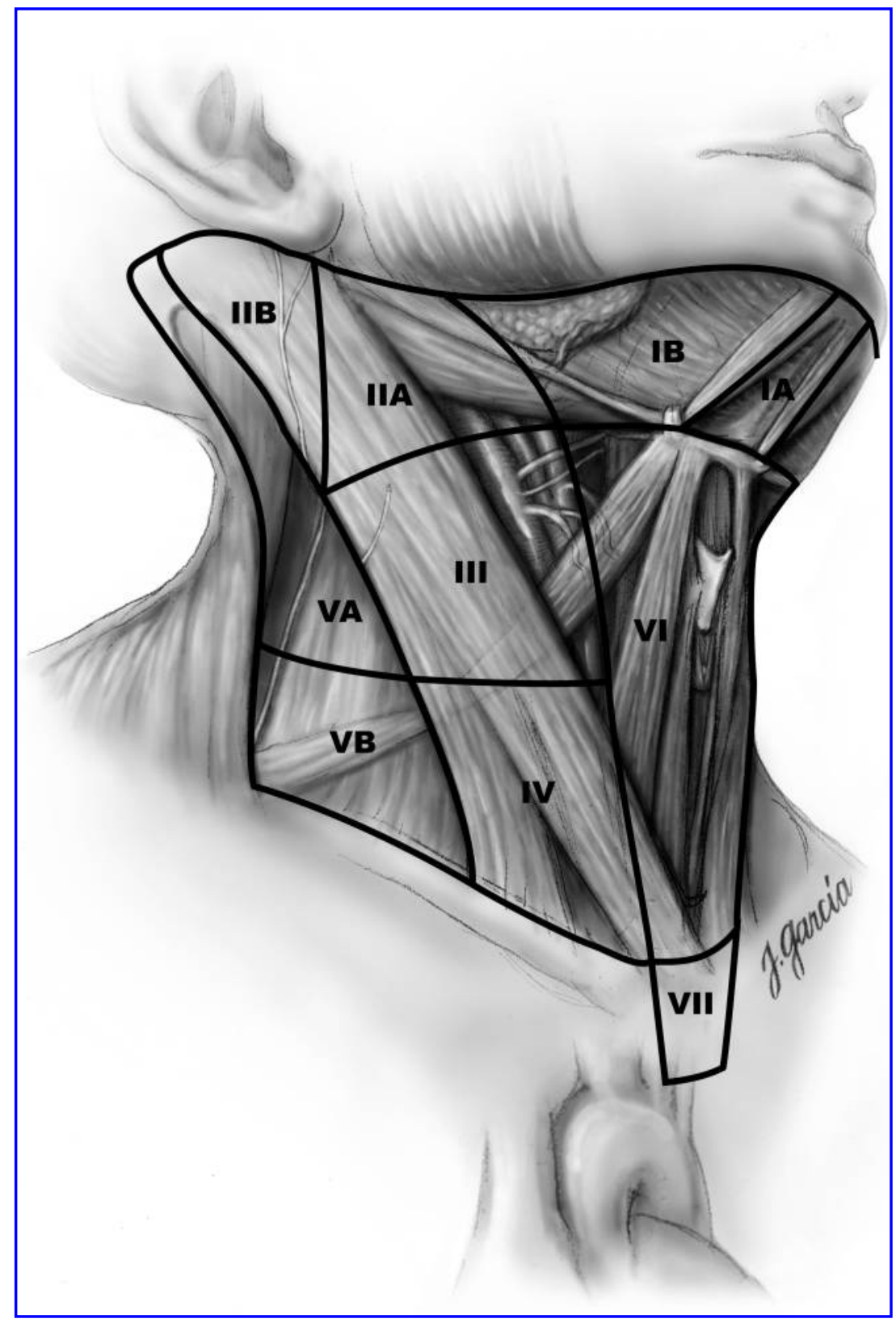

FIG. 1. Schematic right anterior oblique view indicating levels of the neck and upper mediastinum relevant to neck dissection.

\section{Vessels}

The superior thyroid artery is a branch of the external carotid and provides blood supply to the superior pole of the thyroid and occasionally to the superior parathyroid glands via the posterior branch. The inferior thyroid artery is a branch of the thyrocervical trunk, courses posterior to the common carotid, and provides blood supply to the thyroid gland, as well as to the inferior and superior parathyroid glands via inferior and superior branches. The superior, middle, and inferior thyroid veins drain into the internal jugular vein and the inferior veins often directly into the brachiocephalic vein. The thymus lies just ventral to the brachiocephalic vein into which it drains directly.

\section{Nerves}

The external branch of the superior laryngeal nerve comes from the vagus nerve coursing inferiorly along the superior thyroid vasculature to insert into the cricothyroid muscle, an extralaryngeal tensor of the vocal cords. This nerve is identifiable in most cases except when it courses deep into the inferior pharyngeal constrictor and may only be visible within the fascia of the cricothyroid muscle.

The recurrent laryngeal nerve comes from the vagus nerve in the superior mediastinum coursing around the arch of aorta on the left and around the subclavian artery on the right. From there it travels superiorly in the tracheoesophageal groove on the left and somewhat more lateral to medial on the right. 


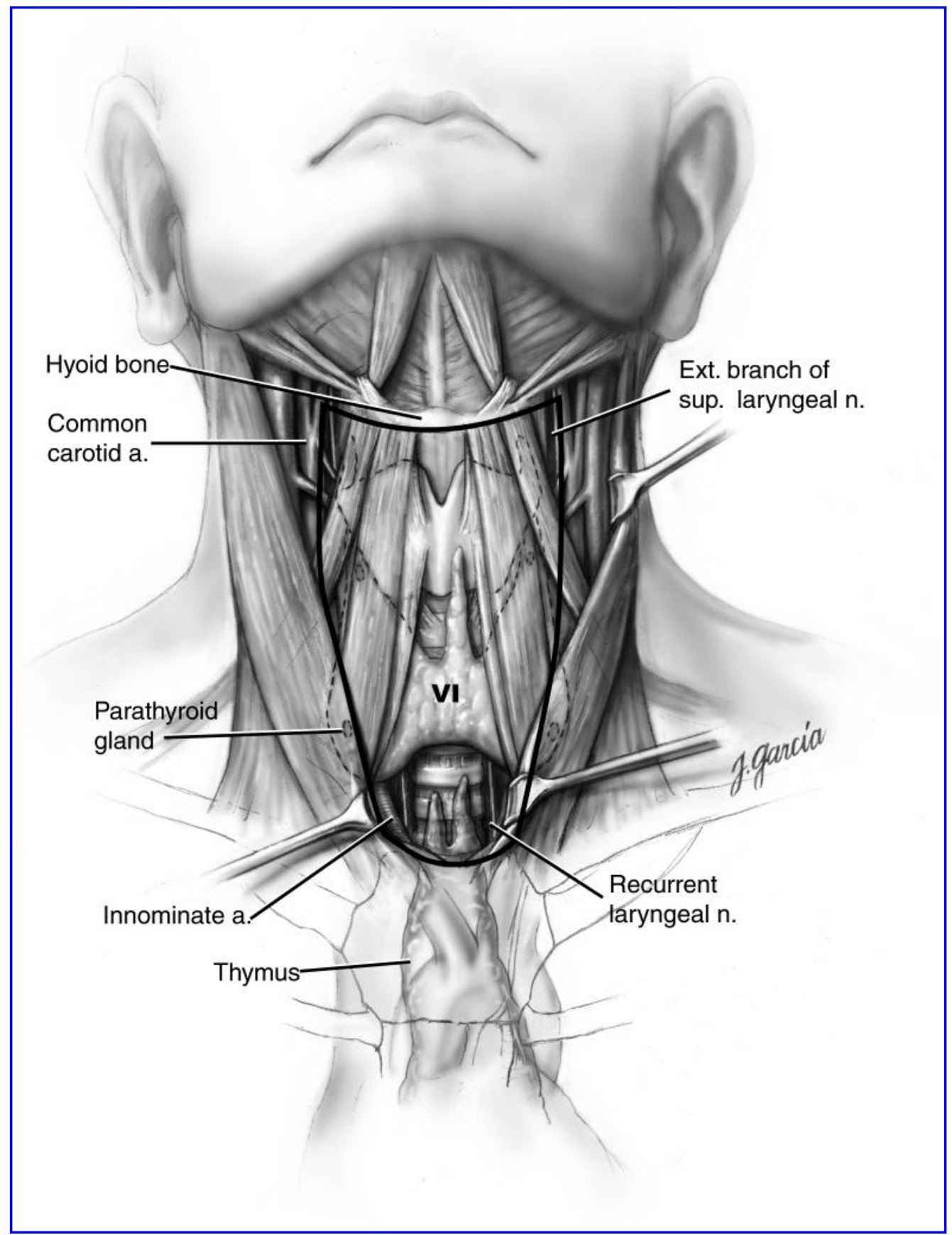

FIG. 2. Schematic anterior view of the neck indicating boundaries of the central neck compartment.

As it ascends it can give off several regional sensory branches to the trachea and esophagus. The recurrent laryngeal nerve motor fibers continue superior and medially in close proximity to the ligament of Berry and then enter the larynx under the inferior pharyngeal constrictor muscle, providing innervation to intrinsic laryngeal muscles (lateral and posterior cricoarytenoid, thyroarytenoid, and interarytenoid).

\section{Parathyroid glands}

The superior parathyroid glands primarily receive blood supply from the inferior thyroid artery via its superior branch, and occasionally from the posterior branch of the superior thyroid artery. The superior parathyroid glands descend from the fourth pharyngeal pouch and most commonly are found just posterior to the recurrent laryngeal nerves near the ligament of Berry. The inferior parathyroid glands descend from the third pharyngeal pouch and are more variable in location but usually are anterior to the recurrent laryngeal nerve near the inferior pole of the thyroid gland. The inferior parathyroid glands receive blood supply from the inferior thyroid artery and are often in close proximity to it.

\section{Terminology}

The literature contains variable terminology relevant to the central neck dissection. The following definitions are suggested for the purpose of consistent terminology usage within 


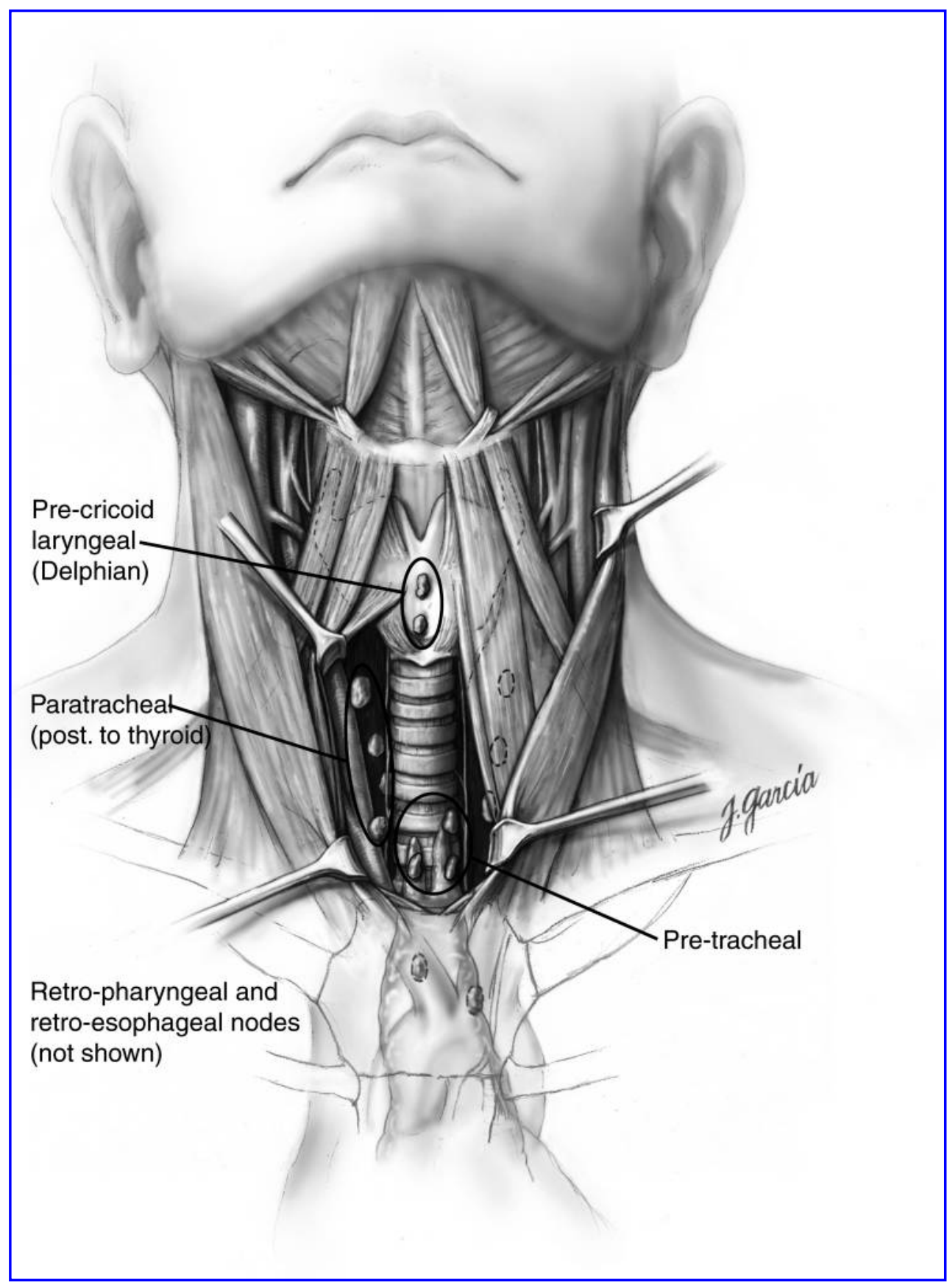

FIG. 3. Detailed anterior view of the central neck compartment indicating locations of lymph node basins relevant to central neck dissection for thyroid carcinoma.

publications regarding the effectiveness and safety of the central neck dissection for thyroid cancer.

\section{Therapeutic versus prophylactic/elective central neck dissection}

A therapeutic central compartment neck dissection implies that nodal metastasis is apparent clinically (preoperatively or intraoperatively) or by imaging (clinically N1a). A prophylactic/elective central compartment dissection implies nodal metastasis is not detected clinically or by imaging (clinically N0). The importance of this distinction when reporting results from studies cannot be overemphasized as the impact of clinically detectable nodal metastasis may differ from microscopic pathologic nodal metastasis. Prophylactic dissection is synonymous with elective dissection.

\section{Describing the extent of central neck dissection}

At a minimum, central compartment neck dissection should include the prelaryngeal, pretracheal, and at least one paratracheal lymph node basin. Lymph node "plucking" or "berry picking" implies removal only of the clinically involved nodes rather than a complete nodal group within the compartment and is not synonymous with a selective compartment-oriented dissection. Isolated removal of only 
grossly involved lymph nodes violates the nodal compartment entered without adequately addressing its disease and may be associated with higher recurrence rates and morbidity from revision surgery (1).

A designation should be made as to whether a unilateral or bilateral paratracheal central neck dissection is performed and the operative record should indicate which side (right or left) in unilateral cases.

- Central neck dissection, bilateral: Removal of the prelaryngeal, pretracheal, and both the right and left paratracheal nodal basins.

- Central neck dissection, unilateral: Removal of the prelaryngeal, pretracheal, and one paratracheal nodal basin.

Central neck dissection may be extended to include comprehensive removal of additional nodal basins including the retropharyngeal, retroesophageal, paralaryngopharyngeal (superior vascular pedicle), and/or superior mediastinal (inferior to innominate artery). A designation should be made as to which additional nodal basins are included in the dissection.

\section{Description of Central Neck Dissection}

A central neck dissection includes removal of the prelaryngeal nodes superiorly and the pretracheal nodes inferiorly to the innominate artery. Also removed are the paratracheal lymph nodes inferiorly from the cricoid cartilage to the innominate artery on the right, and on the left to the axial plane where the innominate crosses the trachea. The recurrent laryngeal nerves are dissected with an atraumatic technique, minimizing manipulation and traction. The superior parathyroid gland is preserved in situ along with its primary blood supply from the superior branch of the inferior thyroid artery. The inferior parathyroid gland is usually reflected laterally along with its blood supply from the inferior thyroid artery. Parathyroid autotransplantation is performed if the glands are devascularized during dissection. Paratracheal tissue specimens are examined for parathyroid glands that may be salvaged and transplanted before being removed from the sterile field. Confirmation of parathyroid tissue with biopsy and frozen section before autotransplantation may reduce the risk of tumor transplantation in the presence of nodal metastasis. Paratracheal nodes are present posterior to the carotid artery on the right, as well as posterior to the inferior thyroid artery and recurrent laryngeal nerve on either side, which may require removal.

\section{Summary}

We have formulated a multidisciplinary consensus statement on the terminology and classification of central neck dissection for thyroid cancer. Central neck dissection at a minimum should consist of removal of the prelaryngeal, pretracheal, and paratracheal lymph nodes (either ipsilateral or bilateral). The description of a central neck dissection should include both the indication (therapeutic vs. prophylactic/elective) and the extent of the dissection (unilateral or bilateral). The use of these common definitions and descriptors will facilitate communication between health care providers and also foster a more uniform reporting of results in clinical studies.

\section{Acknowledgments}

The authors would like to acknowledge Robert Udelsman, M.D., M.B.A., and the artist Juan Garcia, M.A., C.M.I., for the development of these high-quality original illustrations.

The American Thyroid Association Surgery Working Group was chaired by Dr. Doherty and Dr. Tufano.

\section{Disclosure Statement}

GMD is a consultant for MedTronic ENT. RTK has received grant-research support from Genzyme, Bayer-Onyx, Eisai, and Veracyte; is a consultant for Genzyme, Bayer-Onyx, Abbott, and Veracyte; and is on the Speakers Bureau for Genzyme and Abbott. He has received no honoraria for commercial speaking since November 2006 and all commercial consulting since that time has been approved by the ATA Board of Directors, the ATA Ethics Committee, and has been without financial compensation. SJM has received grantresearch support from Veracyte and has been a CME speaker for Genzyme. GWR has been a consultant for Genzyme. DLS has received grant-research support from Veracyte, Wyeth, Astra-Zeneca, and Gyrus. RMT is a consultant for Genzyme, Abbott, and Eli Lily, and has received honoraria from Genzyme and Abbott. SEC, DSC, Q-YD, BCS Jr., DJT, GBT, $\mathrm{RPT}$, and RU report that no competing financial interests exist.

\section{References}

1. Cooper DS, Doherty GM, Haugen BR, Kloos RT, Lee SL, Mandel SJ, Mazzaferri EL, McIver B, Pacini F, Schlumberger M, Sherman SI, Steward DL, Tuttle RM 2009 Revised American Thyroid Association management guidelines for patients with thyroid nodules and differentiated thyroid cancer. Thyroid 19:1167-1214.

2. American Thyroid Association 2009 Peer Guidelines for Physicians. Available at http://thyroid.org/professionals/ publications/guidelines.html. Accessed August 9, 2009.

3. Robbins KT, Medina JE, Wolfe GT, Levine PA, Sessions RB, Pruet GW 1991 Standardizing neck dissection terminology. Official report of the Academy's Committee for Head and Neck Surgery and Oncology. Arch Otolaryngol Head Neck Surg 117:601-605.

4. Robbins KT, Shaha AR, Medina JE, Califano JA, Wolf GT, Ferlito A, Som PM, Day TA 2008 Consensus statement on the classification and terminology of neck dissection: Committee for Neck Dissection Classification, American Head and Neck Society. Arch Otolaryngol Head Neck Surg 134:536-538.

5. Robbins KT, Clayman G, Levine PA, Medina J, Sessions R, Shaha A, Som P, Wolf GT 2002 Neck dissection classification update: revisions proposed by the American Head and Neck Society and the American Academy of OtolaryngologyHead and Neck Surgery. Arch Otolaryngol Head Neck Surg 128:751-758.

Address correspondence to: David L. Steward, M.D. Department of Otolaryngology-Head and Neck Surgery University of Cincinnati College of Medicine 231 Albert B. Sabin Way

Cincinnati, $\mathrm{OH} 45267-0528$

E-mail: david.steward@uc.edu 


\section{This article has been cited by:}

1. Deborah K. Cunningham, Katherine A. Yao, Roderick R. Turner, Frederick R. Singer, Andre R. Herle, Armando E. Giuliano. 2010. Sentinel Lymph Node Biopsy for Papillary Thyroid Cancer: 12 Years of Experience at a Single Institution. Annals of Surgical Oncology 17:11, 2970-2975. [CrossRef]

2. N. Gopalakrishna Iyer, Ashok R. Shaha, Alfio Ferlito, K. Thomas Robbins, Jesus E. Medina, Carl E. Silver, Alessandra Rinaldo, Robert P. Takes, Carlos Suárez, Juan P. Rodrigo, Patrick J. Bradley, Jochen A. Werner. 2010. Delphian node metastasis in head and neck cancers-Oracle or myth?. Journal of Surgical Oncology 102:4, 354-358. [CrossRef]

3. Ralph P. Tufano , Emad Kandil . 2010. Considerations for Personalized Surgery in Patients with Papillary Thyroid CancerConsiderations for Personalized Surgery in Patients with Papillary Thyroid Cancer. Thyroid 20:7, 771-776. [Abstract] [Full Text] [PDF] [PDF Plus]

4. H. Dralle, K. Lorenz, A. Machens, M. Brauckhoff, P. Nguyen Thanh. 2010. Tumortyp- und tumorstadienorientiertes chirurgisches Konzept bei Karzinomen der Schilddrüse. Der Onkologe 16:7, 666-677. [CrossRef]

5. Babita Panigrahi, Sanziana A. Roman, Julie Ann Sosa. 2010. Medullary Thyroid Cancer: Are Practice Patterns in the United States Discordant From American Thyroid Association Guidelines?. Annals of Surgical Oncology 17:6, 1490-1498. [CrossRef]

6. Tina W. F. Yen, Douglas B. Evans. 2010. Do Practice Patterns Reflect Practice Guidelines?. Annals of Surgical Oncology 17:6, 1478-1480. [CrossRef]

7. John H. Yim , Sally E. Carty . 2010. Thyroid Surgery and Surgeons: The Common InterestThyroid Surgery and Surgeons: The Common Interest. Thyroid 20:4, 357-358. [Citation] [Full Text] [PDF] [PDF Plus]

8. Gregory W. Randolph, Elizabeth N. Pearce. 2010. Surgery of Well Differentiated Thyroid Carcinoma: The pendulum swings back in the central neck. Endocrine Practice 1:-1, 1-16. [CrossRef]

9. Mark L. Urken. 2010. The Management of Well Differentiated Thyroid Cancer in 2010. Endocrine Practice 1:-1, 1-29. [CrossRef]

10. David S. Cooper, Gerard M. Doherty, Bryan R. Haugen, Richard T. Kloos, Stephanie L. Lee, Susan J. Mandel, Ernest L. Mazzaferri , Bryan McIver, Furio Pacini, Martin Schlumberger, Steven I. Sherman , David L. Steward, R. Michael Tuttle . 2009. Revised American Thyroid Association Management Guidelines for Patients with Thyroid Nodules and Differentiated Thyroid CancerThe American Thyroid Association (ATA) Guidelines Taskforce on Thyroid Nodules and Differentiated Thyroid CancerRevised American Thyroid Association Management Guidelines for Patients with Thyroid Nodules and Differentiated Thyroid Cancer. Thyroid 19:11, 1167-1214. [Abstract] [Full Text] [PDF] [PDF Plus] 